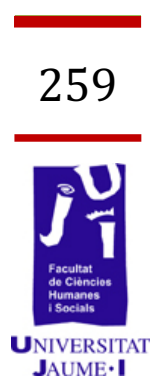

\title{
Cómo funciona un centro de educación especial Todos iguales, todos diferentes
}

\author{
Sara Fernández Membrado \\ al260423@uji.es \\ Adrián Rubio Poveda \\ al225408@uji.es \\ Coral Lorenzo Martí \\ al225303@uji.es \\ Patricia Parrilla Herraiz \\ al228739@uji.es
}


Hace cientos de años, las personas discapacitadas no tenían oportunidad de integrarse en la sociedad, ya que eran abandonadas o asesinadas al nacer. Hubo de pasar mucho tiempo para que tuvieran una oportunidad y, sin embargo, a día de hoy sigue existiendo un cierto rechazo por parte de la sociedad hacia ellas. El problema más grave respecto a la discapacidad es la falta de información sobre esta, incluso por parte de las personas más cercanas a ella. Algunos padres y profesores piensan que los discapacitados nunca tendrán ni autonomía ni futuro. ¿Qué hacer para cambiar esta forma de pensar? ¿Qué medidas tomar para que los discapacitados reciban desde pequeños el trato que se merecen? ¿Cómo conseguir que mejore el conocimiento general sobre el tema? Existen muchas personas y organizaciones dedicadas a dichas tareas. Entre ellas, nos centramos en destacar la labor de los centros de educación especial, que acogen a alumnos cuyas necesidades educativas no pueden verse saciadas en un centro escolar ordinario y les ayudan a integrarse en la sociedad y a vivir con un cierto grado de autonomía. Así pues, en el siguiente trabajo de investigación explicamos con detalle el funcionamiento de estos centros, tomando como referencia el de La Panderola, situado en la ciudad de Vila-real.

Palabras clave: discapacidad, centro de educación especial, necesidades educativas especiales, magisterio, educación, integración.

\section{Introducción}

Nosotros los autores, como futuros docentes, estamos interesados en aprender sobre todo tipo de centros educativos. No queremos dirigir nuestro interés únicamente a un tipo de centro, puesto que en el futuro deberemos estar preparados para dedicarnos a la tarea de educar en cualquier lugar que nos necesite, y por ello decidimos investigar sobre la Educación Especial.

Creemos que es relevante investigar sobre este tema porque mucha gente lo desconoce, y este desconocimiento genera problemas. A día de hoy aún existe el rechazo hacia estas personas. Los centros de educación especial siguen situándose, en muchos casos, en las afueras de las ciudades o pueblos, como una forma de tener a los discapacitados apartados de la sociedad. Algunas personas ni siquiera tienen muy claro a qué se dedican este tipo de centros, y muchos de los padres que llevan allí a sus hijos creen que los centros de educación especial son una especie de guardería en la que los niños pasan el rato sin hacer nada útil. Hay muchísimas clases de discapacidad, y la mayoría de las personas no sabrían distinguir entre apenas dos o tres tipos. Pero más que distinguir entre diferentes clases de discapacidad, se podría decir que el fallo está en no saber cómo tratarlas. Los propios padres de niños discapacitados a veces no parecen tener muy claro qué hacer con sus hijos. El desconocimiento de los padres, y en 
ocasiones de los profesores, llega a un punto que a veces asusta. Esto sucede principalmente porque los padres o tutores legales suelen despreocuparse mucho por sus hijos, con una actitud de no querer mejorar nada, pensando que siempre serán muy dependientes de los demás y que realmente no tienen futuro como trabajadores o en sus relaciones personales. Del mismo modo, también existen padres y tutores que sienten tal obsesión por proteger a sus hijos que no les dejan ni salir de casa ni hacer prácticamente nada por sí mismos. Los esconden de la sociedad. Preocupante es el caso de algunos niños discapacitados, que a los 20 años de edad por primera vez pueden ir solos a hacer tareas tan normales como comprar el pan. Esta sobreprotección ocasiona que el niño no se desarrolle adecuadamente. Necesitan relacionarse con otros niños, necesitan equivocarse para aprender de sus errores y, en resumen, necesitan pasar por todo lo que debería pasar cualquier niño si no queremos que tengan problemas debidos a la falta de contacto con el mundo exterior.

Como se puede comprobar, todavía hay muchos problemas que solucionar respecto al tema, pero en este artículo nos centraremos únicamente en el campo educativo de la educación especial, y más concretamente en el funcionamiento de los centros de educación especial.

\section{Obejtivos}

Conocer las características de un centro de educación especial: encontrar un centro accesible, hablar con el personal del centro, ver las instalaciones, acceder a los archivos de la programación del centro, etc.

\section{Material y método}

El objeto de estudio, ya mencionado anteriormente, fue La Panderola. Es decir, un centro de educación especial situado en la ciudad de Vila-real. Se escogió este centro por su proximidad y porque el personal no puso ninguna objeción a la hora de darnos información. La investigación duró aproximadamente dos semanas, durante las cuales nos informamos sobre la educación especial buscando artículos, mirando la legislación correspondiente a las necesidades educativas especiales, leyendo los documentos educativos de la panderola y haciéndole una entrevista a su director.

\section{Resultados}

\subsection{Procedimiento de escolarización}

Se cita formalmente si un alumno debe acudir a un tipo de centro o a otro a través de un informe psicopedagógico. Sin embargo, ¿qué es lo que se tiene en cuenta para tomar la decisión? La Conselleria de Educación y Ciencia, en la ORDEN de 11 de noviembre de 1994, establece el 
procedimiento de elaboración del dictamen para la escolarización de los alumnos con necesidades educativas especiales. Aludiendo a la LOGSE, este documento determina que la atención al alumnado con necesidades educativas especiales se regirá por los principios de normalización e integración escolar. Es decir, se pretende que la integración de estos alumnos no sea un fin en sí mismo, sino un medio para garantizar la consecución de los objetivos establecidos con carácter general para todo el alumnado. Debido a este hecho, la Ley determina que la escolarización en unidades o centros de educación especial sólo se llevará a cabo cuando las necesidades del alumno no puedan ser atendidas por un centro ordinario. En determinadas circunstancias, cuando las necesidades de los alumnos lo aconsejen, y fundamentalmente para favorecer su proceso de socialización, podrán establecerse fórmulas de escolarización combinadas entre centros ordinarios y de educación especial. Se propondrá la escolarización en el centro de educación especial que corresponda cuando de resultas de la evaluación psicopedagógica se estime que un alumno con necesidades educativas especiales asociadas a discapacidad psíquica, sensorial o motora, graves trastornos del desarrollo y múltiples deficiencias, requiere y requerirá a lo largo de su escolarización adaptaciones curriculares significativas en prácticamente todas las áreas del currículo, o la provisión de medios personales y materiales poco comunes en los centros ordinarios, y cuando se prevea además que en estos centros su adaptación e integración social será reducida.

Así pues, para asegurar la correcta evaluación sobre las necesidades educativas especiales de un alumno, la valoración se llevará a cabo por equipos integrados por profesionales de distintas cualificaciones. Cabe destacar que el dictamen por el cual se determina para un alumno una tipología de centro u otra será reversible y estará sujeto a revisiones periódicas, por si se requiere una modificación del currículum del alumno en base a la evolución de sus características y desarrollo personales. Además, la evaluación psicopedagógica considerará la información relevante para determinar las necesidades educativas especiales del alumno, para la toma de decisiones curriculares y para la propuesta de escolarización, e incluirá información sobre la historia y desarrollo general del alumno, el nivel de competencia curricular y el contexto sociofamiliar.

Por otra parte, las orientaciones específicas para las adaptaciones curriculares comprenderán, al menos, los siguientes aspectos: áreas en las que se requieren adaptaciones curriculares y líneas generales de las mismas; adaptaciones de acceso y los tratamientos especializados y programas específicos que el alumno necesita.

Por último, se realizará el procedimiento para la elaboración y tramitación del dictamen de escolarización para los alumnos de nuevo acceso. Cuando los padres o tutores legales soliciten puesto escolar y manifiesten las características de sus hijos, el director del centro, que será quien reciba la documentación, remitirá la solicitud al servicio psicopedagógico escolar o al gabinete psicopedagógico autorizado correspondiente. Este elaborará el informe técnico de escolarización, que 
será remitido a la Inspección Educativa, que completará el dictamen antes de que se hagan públicas las listas provisionales de alumnos admitidos. El director territorial resolverá a la vista del dictamen y establecerá la ratio que corresponda. La dirección territorial remitirá una copia de la resolución de escolarización a los padres o tutores legales del alumno y otra copia a la dirección del centro donde proceda la escolarización. Durante todo este proceso, los padres o tutores legales del alumno serán informados y consultados de las necesidades educativas especiales, de las opciones de escolarización, de las adaptaciones curriculares y del correspondiente dictamen de escolarización. Además, podrán interponer recurso ordinario contra la resolución de la dirección territorial en el plazo de un mes desde la fecha de notificación.

\subsection{Proyecto curricular de centro}

Al tratarse de un centro de educación especial, el proyecto curricular difiere bastante del de un centro ordinario. En un centro de educación especial como La Panderola, el proyecto curricular de centro cuenta con los siguientes objetivos generales en educación infantil (de 3 a 6 años):

1 - Que los alumnos descubran y valoren su cuerpo, de forma que conozcan sus posibilidades y limitaciones, al mismo tiempo que adquieren una imagen positiva de ellos mismos.

2 - Actuar de manera cada vez más autónoma en sus actividades habituales, para adquirir seguridad y confianza en sí mismos.

3 - Establecer relaciones en su ámbito social para aprender a articular sus intereses y puntos de vista. Establecer vínculos con los adultos que les rodean y con sus iguales.

4 - Aprender observando el entorno que les rodea. Identificar las características y propiedades de los elementos que lo conforman, las relaciones que existen entre ellos y la cultura del lugar en el que viven.

5 - Representar y evocar aspectos diversos de la realidad mediante juegos $u$ otras formas de representación y expresión. Uso del lenguaje verbal de manera ajustada a las diferentes situaciones de comunicación habituales.

6 - Reconocer la existencia de dos lenguas propias de la Comunidad Valenciana y aprender a diferenciarlas, respetando ambos idiomas pero dándole prioridad a la lengua materna.

7 - Enriquecer sus posibilidades expresivas utilizando recursos y técnicas a su abasto y apreciar diversas manifestaciones artísticas.

Para la educación primaria, los objetivos generales seguirán siendo los mismos, aunque se añaden nuevos objetivos de acuerdo a la edad de los niños que se encuentren en este periodo educativo. En infantil y primaria se comparte el objetivo de trabajar el aprendizaje significativo. Es decir, los alumnos adquieren exclusivamente conocimientos muy funcionales y que les puedan ser útiles en su vida diaria. Se busca que aprendan mediante la experimentación y que se sientan motivados haciéndolo. En este tipo de centros, como el cualquier otro, lo que se evalúa es que los alumnos hayan sido capaces de cumplir las expectativas 
de su edad. Sin embargo, no se trata de un sistema que se rige por notas. Se trata de una evaluación contínua que sirve para comprobar si se ha trabajado bien con los alumnos, puesto que el objetivo último es lograr que estos tengan el mayor grado de autonomía posible.

Llegados a la etapa adolescente (educación secundaria) y adulta (transición a la vida adulta o TVA), además de potenciar los objetivos que se habían trabajado hasta entonces también se organizan talleres que les preparan para su posible futuro laboral. Los talleres y la inclusión de los mismos en el currículum es algo que depende de cada centro de educación especial, pero en el caso de La Panderola, los talleres disponibles para los alumnos son los siguientes: encuadernación, informática, cocina, educación vial, huerta y cerámica. Cada taller sigue unos criterios distintos a la hora de evaluar, pero lo que se busca, en líneas generales, es que los alumnos adquieran los conocimientos y capacidades que les permitan ser funcionales en unos trabajos determinados.

Como hemos podido comprobar, se trata de una enseñanza pensada para que los niños con necesidades educativas especiales puedan llegar a desenvolverse en la sociedad, vivir de forma autónoma y tener una calidad de vida tan decente como la de cualquier otra persona.

\subsection{Programación general anual}

Hemos decidido incluír solamente algunos rasgos de la programación general debido a su extensión. Así como hemos hecho con la programación curricular de centro, los ejemplos más extensos serán referentes a la etapa de educación infantil. Comenzaremos nombrando los criterios pedagógicos para la elaboración del horario del alumnado según las diferentes etapas educativas:

- En educación infantil las primeras horas serán sesiones de tutoría, que se dedicarán, en este orden, a trabajar hábitos de autonomía, a realizar las rutinas, al trabajo con fichas y al tiempo de juego dirigido.

- En educación primaria se trabajarán las rutinas y los hábitos de alimentación y autonomía antes de salir al patio. Todo el trabajo que se realiza está globalizado en centros de interés.

- En educación secundaria se trabajarán las rutinas y las distintas áreas instrumentales.

- En TVA (transición a la vida adulta) se hacen las rutinas de manera conjunta y después se divide el grupo en dos. Uno de ellos se va al taller, mientras que el otro se queda en clase trabajando las áreas instrumentales.

- En PQPI la mañana está dedicada en las áreas profesionales.

Después del patio se realizan las siguientes actividades:

- Los alumnos de infantil se van a comer al comedor por cuestiones organizativas. Tienen tres turnos de comedor por falta de espacio.

- Los alumnos de primaria que no tienen autonomía para comer a solas se juntan en el primer turno con el alumnado de infantil.

- El resto de alumnado se reagrupa en grupos flexibles y homogéneos. Aunque a veces los grupos resultantes no se corresponden con los 
grupos del aula, se intenta que el tutor tenga el mayor número de alumnos suyos para poder hacer un seguimiento más directo de sus

avances.

- En la ESO empiezan en los cambios de clases, como sucede en un instituto ordinario, y hacen grupos más homogéneos.

- En TVA, parte de la clase se une a las dinámicas de los PQPI, yendo al comedor y poniendo las mesas, y la otra parte se queda en clase trabajando aspectos más relacionados con la autonomía personal.

En todas las etapas, las tardes (excepto en las situaciones que tengan religión o educación física) están dedicadas a una metodología más dinámica basada en los talleres.

Atendiendo a las características propias de un centro específico, el personal no docente que desarrolla el trabajo de educador tiene unos horarios flexibles para realizar acciones de apoyo a compañeros que necesitan más ayudas para diferentes tareas (cambios de pañales, dar almuerzos o meriendas, etc.). El horario de todo el personal puede estar modificado de manera puntual según las necesidades en momentos concretos como salidas o actividades grupales tanto dentro como fuera del centro.

A continuación, comentaremos la programación general anual en educación infantil.

Las áreas de intervención preferente en educación infantil son las siguientes: autonomía personal, recursos comunitarios, comunicación, motora, habilidades sociales y académico-funcional. Respecto a la metodología que se utiliza, esta se fundamenta en los siguientes conceptos:

- Funcionalidad: el alumno aprende aquello que le da autonomía e independencia respecto al medio y de la dificultad en la que se podría encontrar debido a su discapacidad.

- Actividad: la comprensión del entorno y llegar a transformarlo será posible si el alumno tiene vivencias significativas mediante las cuales va aprendiendo.

- Aprendizaje social: el alumno aprende aquello que debe poner en práctica tanto en grupo como de forma individual.

- Aprendizaje significativo: el alumno aprende aquello que tiene un sentido para él o que le enseñan personas significativas.

- Aprendizaje cognitivo: cuando se produce un hecho que contradiga la hipótesis que el alumno tiene sobre la realidad, este se ve obligado a reestructurar la hipótesis y de hacerla, progresivamente, más ajustada a la realidad.

- Ecología: el alumno aprende conocimientos, actitudes y valores mediante los cuales comprende su entorno vital, se sitúa en él y puede llegar a transformarlo.

Respecto a la temporalización se intentará tener una estructura marcada por hechos significativos (rutinas), dentro de la cual se introducirán variaciones durante la semana. Se cree que algunas rutinas ayudan a que el alumno cree un marco temporal y espacial que le permita 
ubicarse y comprender lo que está ocurriendo y ocurrirá, evitando así un sentimiento de inseguridad e incomprensión de las situaciones. Los agrupamientos tienen que ser flexibles en función del tipo de actividad y éstas deben de ser repetidas diariamente: actividades de autonomía, de comprensión, de expresión, de socialización y de colaboración. Además, también hay actividades más específicas como la fisioterapia y la logopedia que se llevan a cabo si las necesidades educativas de los alumnos lo requieren.

En la etapa de la educación infantil la evaluación que se plantea tiene una evidente función formativa, sin carácter de promoción ni de calificación del alumnado. Una evaluación que pretende mostrar el grado en el cual cada alumno va adquiriendo sus diferentes capacidades, al mismo tiempo que evalúa el proceso de enseñanza y aprendizaje, la práctica docente y el proyecto curricular que se esté desarrollando. La evaluación será global, referida al conjunto de las capacidades expresadas en los objetivos generales de etapa. Estos objetivos, adecuados al contexto del centro y a las características de los alumnos, constituyen los referentes básicos y permanentes de la evaluación. En consecuencia, la evaluación proporcionará una información constante que permita mejorar tanto los procesos como los resultados de la intervención educativa. Un dato importante es que se tendrá un contacto periódico con los padres mediante la entrega de los informes a través de la agenda y de entrevistas al menos una vez al trimestre.

\subsection{Talleres}

Como ya hemos mencionado anteriormente, en los centros de educación especial a menudo se trabaja por proyectos y talleres, ya que la metodología es mucho más práctica que en un centro ordinario. Así pues, se opta por trabajar de este modo contenidos como religión, educación física, artes gráficas y jardinería. Esta metodología por proyectos tiene varios objetivos específicos en común, que tienen que ver especialmente con el mundo laboral.

1. Conocer la utilización de la documentación, entidades y tramites para la búsqueda de ocupación.

2. Sensibilizarse en relación a la importancia de la seguridad y la higiene del trabajo, especialmente en relación al perfil profesional del programa.

3. Conocer a los alumnos y sus motivaciones personales para conseguir los resultados máximos y óptimos y las actividades más adecuadas a su personalidad.

4. Desarrollar en los alumnos todas aquellas habilidades polivalentes que están implícitas en la realización de distintas tareas específicas.

5. Identificar y utilizar adecuadamente los diferentes materiales y herramientas, mostrando respeto hacia el medio ambiente.

6. Adquirir hábitos personales relacionados con el trabajo, que posibilitan y potencian la autonomía laboral. 
7. Desarrollar las actitudes laborales básicas de seguridad e higiene en el trabajo necesarias para el adecuado cumplimiento de una ocupación.

8. Valorar los sentimientos de satisfacción que produce la realización de un trabajo, superando las dificultades y aceptando las limitaciones individuales.

9. Orientar a los alumnos según sus preferencias, características personales y recursos hacia una integración socio-laboral adecuada.

10. Desarrollar estrategias básicas necesarias para la búsqueda de una ocupación y conocer las normas fundamentales de funcionamiento de un lugar de trabajo.

Respecto a los contenidos, encontramos también varios en común:

- Sobre la comunidad: igualdad de sexos, información sobre ellos mismos y sobre los otros, tomar conciencia del valor del trabajo y de los derechos y deberes de la actividad laboral general, normas sociales relacionadas con el mundo del trabajo y uso de recursos comunitarios relacionados con el mundo del trabajo y el ocio.

- Sobre la información laboral: sueldos y nóminas, jornada de trabajo y horas extraordinarias, descanso semanal, fiestas, permisos y vacaciones, faltas y sanciones de los trabajadores, contratos de trabajo y jerarquías dentro de la empresa, características y habilidades propias de algunas ocupaciones y fuentes de información sobre el mundo del trabajo.

- Sobre la solicitud de plaza de trabajo: utilización del teléfono, instancias y otros documentos relacionados para la demanda de faena, fórmulas de presentación, fechas personales, ropa adecuada, aspecto personal y elaboración del currículum.

- Sobre el seguimiento del horario: puntualidad, el problema del absentismo, descanso y cambios de actividad dentro del horario del trabajo.

- Sobre las rutinas de trabajo: conocimiento de itinerarios del centro hacia el lugar de trabajo y cercanías, ropa adecuada a la actividad, desplazamientos, seguimiento de las principales rutinas diarias, normas básicas del proceso de trabajo, cooperación y organización en el trabajo.

- Sobre la seguridad e higiene en el trabajo: normas de seguridad, utilización correcta de equipos de trabajo, higiene postural y mantenimiento de la higiene en el lugar del trabajo.

- Sobre procedimientos para afrontar accidentes, emergencias y situaciones imprevistas: identificación de situaciones de peligro y emergencias, capacidad de reacción, conocimientos de recursos materiales, comunicación a la persona encargada en caso de accidente, emergencias y situaciones imprevistas y primeros auxilios.

- Sobre actitudes respecto al trabajo: concentración, productividad y calidad, motivaciones económicas y personales, aceptación de normas y jerarquías, quejas, conflictos y reivindicaciones lícitas, evaluación y autoevaluación del trabajo realizado y tener conciencia de los progresos.

Por supuesto, en cada proyecto o taller se trabajarán contenidos y metodologías diferentes. Sí que encontraremos similitudes respecto a 
ciertas actividades como fichas, vídeos y trabajos de investigación. Por último cabe destacar que, como habíamos mencionado antes, la evaluación será siempre un proceso de observación por parte de los maestros, continua, formadora y orientadora, teniendo como referencia el progreso propio del alumnos. Todo el personal educativo del centro debe estar implicado en el proceso de adecuación de objetivos, materiales, recursos, metodología y otros factores. Es importante promover una abundante participación del alumnado en este proceso con la introducción de la autoevaluación, proporcionando información al alumno sobre el momento del proceso de aprendizaje en el que se encuentra. La finalidad de la evaluación es orientar el proceso de enseñanza-aprendizaje, mejorar la función docente y evaluar la consecución de los objetivos educativos, capacitando al alumnado para conseguir el aprendizaje y para hacer frente a su futuro cercano.

En cuanto a educación primaria, los objetivos generales de la programación general anual se centran en las áreas académico-funcional, de comunicación, motora y de habilidades sociales. La metodología que utilizarán a lo largo del curso se basa en la relación entre las estructuras psicológicas del alumno y la estructura lógica de los contenidos. Se tomarán como punto de partida los conocimientos previos y se planificarán estrategias que favorezcan la motivación del alumno. Al igual que en el resto de niveles, se garantizará la funcionalidad de los aprendizajes, siendo muy importante que el alumno participe activamente en el proceso de enseñanza-aprendizaje. El profesor debe guiar y estructurar la actividad del alumno, favoreciendo un desarrollo gradual de la autonomía personal y social, atendiendo a la diversidad de cada grupo y a las necesidades particulares de cada alumno, promoviendo metas colectivas que favorezcan el trabajo cooperativo y garantizando la evaluación continua con el objetivo de poder intervenir en el momento adecuado. Es importante considerar la comunicación como base de aprendizaje, desarrollando estrategias de comunicación y utilizando, si es necesario, sistemas alternativos que ayuden a superar los problemas de comunicación y fomenten el desarrollo de las competencias sociales. Deben permitirse agrupaciones flexibles y potenciarse actitudes que favorezcan la igualdad, además de estimular la participación de los padres y madres para potenciar la coordinación y cooperación entre ellos y la escuela.

El trabajo en educación secundaria se desarrolla en diferentes talleres. Para llevarlos a cabo se organizan agrupamientos flexibles de las tres aulas que componen el ciclo. Los contenidos curriculares pueden dividirse en componentes pequeños más asequibles. A los alumnos se les proporciona ayuda física, gestual y verbal, pero en lugar de dársela directamente se demora temporalmente la ayuda, de forma que aprendan a ser más autónomos. Se proporciona motivación extrínseca e intrínseca a la hora de realizar las actividades, partiendo de los conocimientos previos de los alumnos para que el aprendizaje sea más significativo. Se aprenden habilidades funcionales y apropiadas a su edad cronológica, que incluyen 
autocontrol verbal y físico. Las actividades tienen un carácter funcional y están centradas en las necesidades e intereses de los propios alumnos. Se priorizan los aspectos relacionados con el desarrollo de las capacidades motrices, la comunicación y las relaciones sociales. El objetivo es desarrollar al máximo las capacidades de los alumnos y fomentar su participación en ámbitos en los cuales se desarrolla la vida adulta, promoviendo los conocimientos instrumentales básicos y potenciando los hábitos relacionados con la salud corporal, la seguridad personal y el equilibrio afectivo para favorecer y desarrollar su vida con el mayor grado de bienestar posible.

Para finalizar, el aula de TVA está organizada de forma que en las horas de taller acude la mitad de la clase, y la otra parte se queda en el aula de referencia. Esta estructuración se realiza para facilitar el aprendizaje del alumnado y poder así, por parte de los profesionales, interaccionar de una forma más directa e individualizada con los alumnos. Además, TVA cuenta con una hora semanal de educación física, dos de religión, dos de taller de cocina y, junto con secundaria, realizan teatro y piscina cada quince días. La programación general anual está dividida en tres ámbitos: desarrollo personal, desarrollo social y desarrollo laboral. Además, cada uno de los ámbitos potencia las competencias básicas de manera globalizada. Existen objetivos del ámbito de desarrollo personal, de desarrollo social y de desarrollo laboral. La evaluación al principio será diagnóstica para que personal docente se ponga en contacto con los conocimientos previos de los alumnos. También se le dará un carácter formativo, interviniendo sobre cualquier error que se pudiera plantear en el proceso de aprendizaje. Se evaluarán los contenidos programados así como las aptitudes. Además, en ningún momento se limitará el tiempo para conseguir los objetivos de una manera rigurosa. En cuanto a la metodología, los diferentes ámbitos de experiencia tendrán un carácter abierto y flexible, de forma que puedan asegurarse la generalización de los aprendizajes relativos a las rutinas y a las diferentes tareas. Los aprendizajes, y en especial la lectura y escritura, deberán orientarse hacia la consecución de los siguientes campos de habilidades: lectura relacionada con las actividades laborales, lectura relacionada con la planificación de las actividades diarias, lectura o identificación de imágenes de recetas de cocina, identificación de etiquetas en los productos de un supermercado y cálculo centrado fundamentalmente en el uso del dinero en las situaciones y actividades cotidianas. En líneas generales, se tendrá presente al desarrollar las actividades la heterogeneidad madurativa que presentan los alumnos y la consonancia con sus intereses, factores que suponen introducir materiales individualizados. Asimismo, se evaluará la programación del docente y el desarrollo real de los programas en relación con su adecuación a las necesidades educativas específicas del alumnado, teniendo en cuenta las aportaciones del resto de los profesionales del centro. En cuanto a la evaluación del alumnado, esta se llevará a cabo con la referencia de los objetivos y criterios de evaluación establecidos para cada alumno de TVA. Al finalizar cada año, el tutor, en colaboración con el resto de profesionales, 
elaborará un informe escrito sobre el progreso de cada alumno, que se adjuntará al expediente del alumno. Además, se realizará una evaluación inicial a comienzo del curso, otra en enero y otra al finalizar el curso.

\subsection{Personal del centro}

El centro de educación especial La Panderola cuenta actualmente con un equipo de 29 profesionales encargados tanto de la enseñanza como del cuidado de los niños. El personal del centro se divide en distintos tipos de profesores, aunque no hay tanta diversidad como en un centro ordinario. Hay que tener en cuenta que no se estudian asignaturas ordinarias, ya que el objetivo primordial de los profesores es el de conseguir que los alumnos aprendan a valerse por sí mismos en la medida de los posible. Además, si se quiere que los niños aprendan ciertas cosas sobre el mundo que les rodea, habrá una gran tendencia a que las aprenderán de forma práctica, y esto se demuestra en las numerosas salidas al exterior que se llevan a cabo.

Aunque no se impartan asignaturas propiamente dichas, algunos profesores están especializados en música, lengua o religión, pero no enseñan dichas materias como si estuvieran en un centro ordinario. Por ejemplo se puede llevar a cabo un plan de normalización lingüística, pero debido a las características de los alumnos, un plan plurilingüe estaría fuera de lugar.

Además de profesores, a un centro de educación especial le va bien disponer de personal médico, aunque no siempre puede tenerlo. Un centro ordinario no necesita a personas especializadas en medicina como las que pueden encontrarse en un centro de educación especial. Este personal médico no solo es útil por lo que les pueda pasar a los alumnos o por estudiar su evolución, sino también porque en ocasiones los padres no conocen ciertos aspectos sobre la condición de sus hijos

\subsection{Instalaciones}

El centro de Educación Especial la Panderola cuenta con las siguientes instalaciones: despacho de dirección, despacho de secretaría, aula TVA, sala de profesores, 3 aulas de logopedia, aula de infantil, despacho de prefectura de estudios y fotovídeo, 2 aulas de PQPI, despacho del psicopedagogo, 3 aulas de ESO, sala multifuncional, 3 aulas de primaria, archivo, biblioteca, sala de fisioterapia y sala DUE, cocina, comedor y taller.

Las instalaciones se asemejan bastante a las de un centro ordinario, con la diferencia de que los centros de Educación Especial tienen varias aulas que sirven específicamente para llevar a cabo una metodología que se adapte a las Necesidades Educativas Especiales de los alumnos. Por otra parte, poseen también una serie de recursos materiales como sillas de 
ruedas y una especie de camilla para que los niños con disfunciones motoras puedan cambiar de postura.

\subsection{Alumnado con necesidades educativas especiales por sobredotación intelectual}

Consideramos importante mencionar a los alumnos con sobredotación intelectual, que no asisten a los mismos centros especiales que el resto de alumnos con necesidades educativas especiales. Estos pueden asistir a centros especializados para superdotados o estar en una aula ordinaria. La ley indica que se debe proporcionar a todos los alumnos una educación adecuada a sus características y posibilidades. Se considera que los niños que sobresalen de los demás por su capacidad, denominados por la LOE como alumnos de altas capacidades intelectuales, son englobados en el grupo de alumnos con necesidades educativas especiales. Existe la posibilidad y la necesidad de atender a aquellos alumnos que sobresalen o tienen posibilidades de sobresalir. Tienen el mismo derecho que los demás de ser atendidos para desarrollar todas sus capacidades. Se trata de detectar cuáles son sus capacidades y posibilidades, y de responder a su potencial. Hasta estos momentos se ha puesto todo el esfuerzo en atender a aquellos alumnos con dificultades en el aprendizaje, dejando de lado en muchas ocasiones a aquellos alumnos que tienen un gran potencial. Desde la LOGSE se ha incorporado la necesidad de la atención a estos alumnos, pero apenas se ha desarrollado legislación específica y, mucho menos, programas de actuación. Así pues, es necesario crearla, pero desde una perspectiva inclusiva, procurando no tratarlos como a un grupo diferente, sin excluirlos de la normalidad de una clase. Necesitan una atención y unos recursos determinados. No se puede desperdiciar la reserva de talento en una sociedad que se caracteriza por el valor creciente que adquieren la información y el conocimiento para el desarrollo económico y social.

- Tópicos y realidades: cuando se habla de estos alumnos, rápidamente vienen a la mente unas características que se les atribuyen de forma habitual. Se piensa que son alumnos con dificultades de socialización, de adaptación, de integración, marginados, con problemas en clase, etc. No dejan de ser tópicos, en algunas ocasiones con visos de realidad. Podemos apreciar tres características bastante comunes en estos alumnos, provocadas más bien por la falta de atención adecuada que por sus propias capacidades. En primer lugar, una tendencia al aburrimiento en clase, que conlleva desmotivación y, a veces, conductas disruptivas en detrimento del rendimiento académico y las relaciones con el profesor. El alumno superdotado se aburre en clase porque no le interesa nada lo que le proponen. Por lo tanto, el origen del problema está en la propuesta de trabajo y no en su propia manera de ser. En segundo lugar, también existen ciertas dificultades de socialización en algunos casos, sobre todo en aquellos en los que hay poca flexibilidad, una cierta introversión y mucha presión del ambiente familiar. Finalmente, se pueden desarrollar conflictos personales y una cierta falta de seguridad, ya que muchas veces se olvida 
que un niño de altas capacidades sigue siendo un niño con emociones e intereses propios de su edad.

- Actitudes que hay que tener en cuenta en la relación con los alumnos: las siguientes actitudes se pueden aplicar a cualquier alumno, tenga necesidades educativas especiales o no. Aceptarlos como son, teniendo siempre presente que son, en primer lugar, niños; estimularlos sin forzarlos ni agobiarlos; dejarles pensar por su cuenta y proteger el poder creativo que tienen; ser flexibles y respetuosos con su trabajo y tratar de participar de sus inquietudes, animándoles a resolver sus problemas sin temor al fracaso y ayudándoles a la planificación de sus proyectos y tareas. Y todo esto siempre desde una actitud de acompañamiento del profesor. Este planteamiento choca frontalmente con la estructuración de nuestros centros y con el currículo que tienen que desarrollar. Los centros educativos están organizados de tal modo que contamos con unos espacios de aprendizaje en los que un grupo de 30 niños debe desarrollar el mismo currículo, conseguir unos resultados previstos y hacerlo en un tiempo previsto igual para todos. Los objetivos están planeados por la administración. Lo que proponemos es dejar de pensar en programas específicos para aquellos niños que tienen una discapacidad determinada y para los que disponen de un talento especial y plantear clases en las que la diversidad sea realmente una realidad. Se trata de pensar en qué es lo que necesitan nuestros alumnos, qué posibilidades tienen, qué dificultades pueden encontrar, y diseñar procesos adecuados a cada uno de ellos, en el marco de una clase que va trabajando de una forma natural y colaborativa como un grupo real de trabajo.

- El centro en su globalidad para una enseñanza inclusiva: algunos países, entre ellos España, se mueven alrededor de tres tipos de medidas. En primer lugar, lo que denomina una enseñanza diferenciada, ya sea como un grupo concreto en la propia clase constituyendo grupos específicos en el centro, desarrollando un currículo enriquecido y diferenciado. La segunda medida se basa en una atención individualizada en función de las necesidades y características de cada alumno, es lo que llamamos enriquecimiento. Finalmente, la aceleración -adelantar uno o más cursoses la medida que se ha aplicado con más asiduidad en la mayoría de los países, porque es la que implica el menor coste económico para la administración y escasos problemas administrativos y de recursos para el centro.

\section{Discusión y conclusiones}

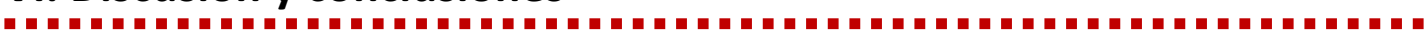

- La sociedad en general no tiene suficiente información sobre los centros de educación especial, y tampoco sobre los alumnos con necesidades educativas especiales.

- En los centros de educación especial, y en cualquier tipo de centro, está muy presente la burocracia. Prácticamente cualquier acción se tiene que registrar en papel. El director de la Panderola afirmó que este hecho 
era agobiante, y que echaba de menos pasar horas como docente con los alumnos.

- Con una visita en primera persona a un centro de esta tipología, hemos aprendido mucho más que con los apuntes teóricos y las leyes referentes a este tema. Hemos visto la realidad del centro en primera mano.

- El director nos comentó también que, debido al desconocimiento de los inspectores de educación sobre necesidades educativas especiales, estos no se inmiscuían demasiado en el funcionamiento del centro.

- Existe un AMPA en el centro, pero más por necesidad de los maestros que de los padres. Esto se debe a que los maestros intentan luchar para que los padres se impliquen en la educación, pero la participación de los padres en el consejo escolar va disminuyendo y actualmente es de un $12,1 \%$. Según el director, los padres no se interesan porque el sistema no está todo lo bien organizado que debería.

- El currículum está hecho por el mismo centro, debido a las numerosas adaptaciones que se deberían hacer si se partiera del currículum original.

- Los alumnos de este centro no utilizan libros de texto, ni los necesitan. Normalmente les basta con llevar una simple libreta con la que tomar apuntes cuando sea necesario.

- El trabajo de campo está muy valorado en un centro de educación especial. Los niños aprenden más cosas saliendo al campo que quedándose en clase. También se valora que distintos tipos de profesionales (policías, bomberos, etc.) acudan al centro para explicar su trabajo a los alumnos.

- En este centro se agrupa a los alumnos por edades, no por tipos de deficiencias. Esto es así porque aunque tengan el mismo nombre, las deficiencias son diferentes en cada persona. Además, en el centro tienden a formar grupos heterogéneos para que los alumnos aprendan los unos de los otros. Como ejemplo, planteamos el caso de poner a todos los alumnos sordos en un aula, lo cual no tendría sentido. Sin embargo, si en la misma aula hay un sordo, un mudo y un ciego estos se podrían ayudar entre ellos.

- Hay diferentes tipos de centros de educación especial. Por ejemplo, en La Panderola se acepta a alumnos con un nivel de discapacidad de leve a moderado. Sin embargo, existen centros que aceptan a alumnos con discapacidades más graves, y centros específicos para alumnos con necesidades especiales derivadas de sobredotación intelectual.

- Es la administración, con la colaboración de profesionales en educación, la que dictamina a qué tipo de centro deben acudir los alumnos.

- En La Panderola se realizan eventos relacionados con las distintas festividades que ocurren a lo largo del año. Por ejemplo, cuando llega la navidad se monta un belén que se sitúa en la entrada del centro.

- Normalmente, los alumnos llegan a este tipo de centro con tres años de edad, y deben permanecer en él hasta los 21 años a no ser que se queden en el PQPI, donde se les prepara para ejercer un oficio. Para ello se requiere que los alumnos tengan una cierta autonomía. Si la tienen, se les 
orienta hacia un centro profesional. Si no, las familias los llevan a un centro de día. Por desgracia, estos últimos están bastante llenos.

- El centro pide los recursos a conserjería.

- No hay asignaturas y las áreas de trabajo no están delimitadas. Existen cinco áreas básicas: motora, académico-funcional, de recursos de la comunidad y laboral.

- No hay un reglamento orgánico funcional del centro.

- Hay muchos menos alumnos por maestro que en un centro

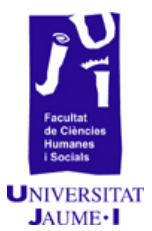
ordinario. En La Panderola hay cinco alumnos por cada maestro. Cada aula tiene un maestro y un educador, y el centro dispone de mucho más personal específico.

- Cada alumno resuelve los problemas a su nivel, y se suele trabajar por proyectos.

- La tarea de los profesores es que los alumnos desarrollen al menos sus capacidades básicas. El $70 \%$ del tiempo lo dedican a enseñarles a ser autónomos.

- Los maestros no están específicamente formados para estar en un centro de educación especial.

- Se organizan salidas que llevan a la práctica los contenidos que los alumnos trabajan en clase teóricamente.

- Al mismo centro pueden ir alumnos de otras localidades cercanas, ya que no hay tantos centros de educación especial como centros ordinarios. Hay muchos alumnos que son transportados desde otras localidades, y se quedan en el centro a comer. Transporte y comida están subvencionados por conserjería.

- No hay una evaluación numérica hacia los alumnos. En las reuniones de ciclo, los profesionales se juntan y evalúan factores como la capacidad de trabajo, los avances, la autonomía, el nivel lecto-escritor-funcional, etc. Se hace un pequeño informe escrito y se comunica a los padres. Se valoran, entre otras cosas, el esfuerzo, la implicación y las ganas de mejorar.

- A los padres se les dice lo positivo, en lo que han mejorado los alumnos y también cómo pueden convivir con sus hijos. Se les pone en la realidad de la discapacidad, y si las estrategias propuestas no funcionan se habla de cambios.

- Se hace una escuela de padres, tratando distintos temas, pero eso no da la garantía de que estos pongan en práctica las propuestas. 


\section{Bibliografía}

VII. Bibliografia

BERNAL, J.L. y T. GAYÁN (2011): "Alumnado con alto rendimiento», Cuadernos de pedagogía, 409, página 52.

BOE núm. 47. ORDEN de 14 de febrero de 1996 por la que se regula el procedimiento para la realización de la evaluación psicopedagógica y el dictamen de escolarización y se establecen los criterios para la escolarización

MARTí SOler, L. (2012): "Abrir ventanas a la discapacidad», Cuadernos de pedagogía, 420, página 14.

ORDEN de 15 de mayo de 2006, de la Conselleria de Cultura, Educación y Deporte, por la que establece el modelo de informe psicopedagógico y el procedimiento de formalización. [2006/6370]

ORDEN de 11 de noviembre de 1994, de la Conselleria de Educación y Ciencia, por la que se establece el procedimiento de elaboración del dictamen para la escolarización de los alumnos con necesidades educativas especiales. [94/8854]

Programación de Actividades auxiliares en víveres, jardines y centros de jardinería del CPEE la Panderola. Curso 2011-2012.

Programas de cualificación profesional inicial del CPEE la Panderola. Curso 2011-2012.

Programación de Educación Física del CPEE la Panderola. Curso 2011-2012.

Programación de Religión del CPEE la Panderola. Curso 2011-2012.

Programación de Taller de Artes Gráficas del CPEE la Panderola. Curso 20112012.

Proyecto Curricular de Centro del CPEE la Panderola. Curso 2011-2012.

Proyecto Educativo de Centro del CPEE la Panderola. Curso 2011-2012.

Proyecto General Anual de Centro del CPEE la Panderola.Curso 2011-2012. 